\title{
5 'Hacking' Life Itself - In Pursuit of a Definition
}

Type 'hacker' into the Google image search and the first hits show a shady figure in a black hoodie, faceless, anonymous, crouched over a computer, with streams of digits in the background. The figure of the hacker - a prominent one in our digital, data-based societies - carries with it a dark connotation: secretive, unofficial, invisible, avantgarde. Type in 'life' and you find images of sunrises and scenery, success, happiness, people and plants. Life is inherently positive. The term 'biohacking' often used for activities that 'hack' life itself, is a perfect amalgamation of these contradictions: the Greek bios, meaning life, becomes a prefix for the hack: 'biohacking,' it seems, is also 'lifehacking.' So how can this 'dark' activity be applied to something connotated so positively? As the previous discussions have shown, this culturally pervasive image of the hacker only carries with it the shady connotations of the (illegal) 'hack:' breaking rules and laws, posing a danger to safety and society, stealing (intellectual) property, entering digital systems uninvited and undetected with malicious intents. ${ }^{1}$ But hacking as an approach is much more diverse: As I have pointed out in the previous chapter, it ranges from these illicit activities, to the coding of free and accessible software, activism for more access to computers and technology or simply an ideology of openness, inclusion, innovation and transparency. In their essence, hacks, according to Delgado and Callen, are experiments that "show that problems can be solved and that things could be done otherwise." They are an "experimental mode of inquiry" in which not the success of making them work counts but the process of trying (189). Today, the object that is hacked does not have to be code: technology, bodies, materials, feelings, lifestyles can be subject to this type of experimental inquiry and transformation. Hacking has left the realm of the virtual, digits and code and instead entered the physical world. In fact, in DIY biology

1 Interestingly, this illegality might come into effect in biohacking in cases where life has been patented: substances such as adrenaline, insulin and vitamin B12 count among the historical examples of biological patents, whereas more recently DNA sequences have caused new debates about the eligibility of biological materials for patents. The most prominent example in genetics is Myriad Cenetics' patent claim for BRCA1, one of the 'breast cancer genes.' As it was common in the early 2000 s, Myriad Cenetics wanted to patent its prestigious finding. This decision received a lot of public backlash, leading to a U.S. Supreme Court decision in 2013 that ruled naturally occurring DNA sequences ineligible for patenting. 
and medicine, hacking is inherently material, focused on tangible technologies, human bodies and biological matter. And more than that, it becomes an activity in which the individual pulls all the strings.

This third part sets out to analyze the different forms that DIY practices take in the context of medicine and biology. Proceeding in the form of case studies, it will span a wide range of techniques and cultural representations, taking up the diversity inherent in this new field of experimentation, knowledge production and public participation. Its diversity, ambivalence and novelty have so far made it hard to find a definition for what 'DIY biology' is, which practices, people and discourses are included, which are excluded. In fact, I would argue that we do not even need such a definition as the (intentionally) unstructured boundaries, its constant shifting and the rapid advancements of the technologies used would obliterate any attempts at definitions before they reach print. $^{2}$ Still, for the sake of my discussion and the sanity of the reader, I will attempt to disentangle some of the practices. Consider the following: 'Body hacking' is often also called 'biohacking,' but 'biohacking' is also a commonly used term for 'DIY biology.' Are those practices one and the same? In truth, they could be considered different practices in the objects they choose to experiment with, but they are related in their core ideas. Many of the terms seem to be used interchangeably both by practitioners and in the public discourses around them, so I will try to somehow differentiate how they can be thought apart and delineate the (open) boundaries that I have set.

Let us start where (presumably) no solid research but all private information endeavors start. ${ }^{3}$ If you look "biohacking" up on Wikipedia, you will land on a disambiguation page, signaling that this title would be suitable for many different pages. The information you find includes the German streaming television series Biohackers as well as links to the following pages:

"- Body hacking, the application of the hacker ethic to improve one's own body

- Do-it-yourself biology, movement in which individuals and small organizations study biology

- Quantified self, measuring various biomarkers and behaviors to try to optimize health

- Performance Psychology, improving ones mental and behavioural capabilities to boost performance"

(Wikipedia, Biohacking, 16. April 2021)

Further articles link to Nootropics, Nutrigenomics, and self-experimentation in medicine. A snowball inquiry into the links reveals that body hacking is said to refer

2 In fact, it shares this complexity with attempts at a 'definition' of DIY: The multiplicity of contexts on which the term is used, as I have shown in Ch. 4, means that there is no clear-cut definition of what exactly DIY is.

3 I would argue that Wikipedia as an information source is much more revealing about what public opinion perceives DIY biology to be than any attempts at formal academic elucidation. As an open knowledge base - contributed to by its users - Wikipedia has become the new online encyclopedia used by many people to gather preliminary information. While it is true that Wikipedia articles are susceptible to false or misleading information or plagiarism, they nonetheless reflect what the 'crowd' thinks about the meaning and definition of certain terms. 
primarily to practices of grinding, the biopunk movement and do-it-yourself body enhancements (linking Neurohacking, brain hacking, as a further reference page). The Wikipedia page for "Do-it-yourself biology" lists bioinformatics, genetic engineering, medicine, implants, and art as potential research topics, with community, open source and advocacy being important aspects in this research. "Do-it-yourself biology (DIY biology, DIY bio) [...] as a growing biotechnological movement," the entry reads, can be done for profit or open science innovation, as a personal hobby or community learning effort, in which often people with training mentor those without (Wikipedia, "Do-ItYourself Biology"). All these pages, as Wikipedia and its contributors highlight through the disambiguation page for "biohacking," are somewhat related, almost impossible to differentiate - also linguistically, as many of these terms are used interchangeably. The Wikipedia entries demonstrate how the public conception of DIY biology is characterized by a confusing and complex diversity of its subjects, practices and "related but distinct" sub-movements (and names for these movements) (Wikipedia, "Do-It-Yourself Biology"). What they agree on, however, is the importance of a hacker ethic and hacker culture as well as the associated values.

In 2015, Nootka St. Film Company, a small creative studio based in Canada, released a short documentary on YouTube titled: SHIFT: Biohacking Documentary. ${ }^{4}$ Part of their SHIFT online documentary series, this 13-minute video shows the two directors, producers and presenters Steve Adams and Sean Horlor trying to elucidate what biohacking is by talking to some of its practitioners. What makes this documentary interesting is their inclusion of several different attempts at a concise definition, reflecting the different types of definitions present in the cultural imagination. At the very beginning of the documentary the directors emphasize improvement as the end goal of biohacking: "Biohacking is a growing global movement where anyone, not just scientists, can use technology to improve the human body." For them, the essence of biohacking is the use of technology to enhance our bodies (Adams and Horlor). Another definition provided in the documentary by futurist Nik Badminton focuses on the diversity of the community and its approaches as the main characteristic:

It's very much a self-starting sort of alternative community that believes that as humans we could be so much more. That could be through nutrition, exercise, and diet, and even more extreme forms by ingesting all sorts of drugs, smart drugs, augmenting the body using technology, implanting the body with things. Biohackers are a little less planned and research and a lot more: Let's experiment. Let's see where we can go. (Adams and Horlor, 02:12-02:35). an entry into body augmentation with technology; implants, nanobots, exoskeletons as technologies with medical and rehabilitative potential; as well as genetic tests as a basic form of biohacking - using the gathered data to change habits before they become health concerns. A voice over also acknowledges that not all biohacking is concerned with modifying the human body, but can also study non-human beings as well (Adams and Horlor). The documentary has had 51.184 viewers up until 16.04.2021. 
While it still maintains human improvement as the end goal this definition also underlines the creative, experimental approach. The third definition, given by Amal Graafstra, once more underscores the diversity of the movement: "Biohacking is a big umbrella term. It involves everything from hacking DNA and bacteria and people to doing what we're doing which is essentially not shying away from putting devices into the body to enhance it" (Adams and Horlor, 08:51-09:04). Already this short excursion demonstrates that there is a variety of definitions out there in the public discussion and imagination, many of which include human improvement, interaction with technology and an experimental ethos. ${ }^{5}$

In light of this variety, I have chosen to include all those practices in the area of medicine and biology that are carried out by individuals, ideally out of their own free volition, that follow the ethos of do-it-yourself. Like Graafstra proposes I use DIY biology and biohacking as umbrella terms, which also entails that I too use them interchangeably as denominators for an overarching phenomenon. For me, this includes DIYbio as a social movement and all the practices that entails (from technologies, molecular biology, to art), grinding (implantable technology), body hacking (improving the body) but also commercialized solutions (such as genetic tests, fitness tracking, and so forth). What do all these seemingly disparate techniques have in common? These practices, in my view, are united by their experimental and creative (out-of-the-box) approaches, solution-based and goal-oriented thinking, self-directed and self-imposed nature, and open and accessible character. I explicitly include commercial DIY solutions for the simple reason that they to a large degree follow the same logics of self-responsibility and choice but are more widely available to a broader audience. I consider them a different expression of the same phenomenon. In 2001, Robert Carlson had predicted that biotechnology would move from institutionalized labs to small companies and eventually domesticated spaces like kitchens and garages (Keulartz and van den Belt 2). The small companies that constitute some of the DTC market could thus also be considered a 'way station' between institutionalized science and complete DIY techniques. Moreover, Keulartz and van den Belt claim that grassroots entrepreneurship is a common outcome of DIY biology in the US: Hacker-inventors use the paradigms of DIY bio and the publics' willingness to participate in order to found start-ups (17). Some of the case studies in my discussion show examples of this type of economic activity around the DIY ethos, such as Graafstra's biohacking company Dangerous Things (Case Study VIII). On an even broader scale, commercialized solutions could be considered as an inherent and to a certain degree required part of DIY approaches in health care. In countries like the US, with a focus on prevention and active inclusion of patients into their healthcare, Andelfinger and Hänisch argue, commercial DIY forms, such as webpages, tracking, DTC testing, apps, and gadgets, become increasingly important for health care provision ("Einleitung" 2). In a consumption-oriented society, the consumerist approach of DTC and commercial DIY practices might be a more appealing and accessible way to support those paradigms and partake in their perpetuation.

5 Academic considerations often shy away from a concise definition as well. Instead, the diversity of approaches, ideologies, spaces and practices is demonstrated, while carving out some of the common characteristics. An overview is provided in Chapter 4.2. 
What most of the DIY practices share is that they do not only have cultural, social or political effects but also very much material ones: Some modulate biological materials, others inscribe themselves on bodies and leave their traces, and still others aim at a more profound knowledge of biology (one's own and more generally) in order to use it for individual goals and projects -but they all to some degree affect biological matter. For example, Delgado argues that practices of DIY medicine turn "the body into a test field for medical innovation:" "people are actively using their bodies for producing new diagnosis and for testing treatments," not just when established practices provide no hope, as Delgado claims, but also when they are perceived as insufficiently addressing individual needs (71). The body becomes a test field for experimentation with all the material effects that can entail. Talking to different DIYbio members, Delfanti found that for some the political commitment of DIY also encompasses a more "proactive" attitude to an individual's own biomedical information, facilitated by access and transparency in biological practices (Biohackers 123).

Information about the body is perceived as encoded in its materiality; DIY practices can uncover this information and make it actionable for the self. The human body and its biology become a new type of imagined and material 'frontier,' a new territory full of possibilities that waits to be explored and conquered using the latest technologies. "Jamming stuff into your body, merging machines with your nerves and brain, it's brand new,' said [biohacker] Warwick. 'It's like this last, unexplored continent staring us in the face" (Popper). The body, in biohacking, becomes a charted, but unconquered territory ready for exploration, cultivation, and indeed exploitation. The increased attention towards the material reality of one's body creates a heightened self-awareness of the body and helps modulate the self accordingly. Bodily plasticity is a precondition for this process. The DIY techniques assume the body to be open to change on molecular and molar levels, be it through supplementation, extraction or integration: Molecular pathways are optimized, bodily fluids extracted and analyzed, technologies integrated into the body. All those either open up the body, quite literally, or leave their marks and traces. The entanglement of biomedical knowledge, technology and everyday life alters "how the body is constructed, seen, and talked about," mostly as a sphere that individuals have to shape and improve (Burri and Dumit, "Introduction" 5). Intimate knowledge is often coded as leading to some sort of self-improvement.

The social, cultural, political effects of biohacking are more abstract, concerned with how the self is conducted in society, how techniques are culturally perceived and sanctioned, their future applications and broader implications. I will come to those in the next part of the book, when I take a step back from the case studies to reflect on common themes and discourses, political claims and social consequences. For now, suffice it to mention that some of the core concerns will be questions of participation and literacy, changing roles of power, competence and expertise, feelings of uncertainty and insecurity as well as the repercussions of individualism for society at large. Even smallscale adoptions, like those discussed in my case studies, can further a process of normalization and routinization and thus a transferal of concepts to larger projects. For example, if vitamin supplements or fitness trackers are normalized, interventions using smart drugs or implantable monitors might be considered as the logical, necessary and sanctioned next step. DIY techniques create social change precisely through such 
decisions about their use - about how to use them, when to use them, who can use them or who has to use them (cf. Brodwin 3). These decisions are embedded in the personal and political stakes that individuals and societies have in them.

\section{Practices and Representations}

How do I approach my case studies in this chapter to elucidate both the concrete material effects of the practices as well as their social, cultural and political implications? In this part, I access those material practices primarily, but not exclusively, through representations, meanings and discourses. Probably more so than the practices themselves, cultural representations shape public discussions and can shine a light on how we talk about these practices, which hopes and fears are embedded into them, how they shape lives and bodies, and how they are publicly evaluated. ${ }^{6}$ My goal is to describe the relationship of individual 'texts,' broadly defined, to the cultural and biological imaginaries of the audience and producers of the texts and to elicit the values, discourses and politics inscribed into the texts - to show the 'cultural work' these texts do. The representations under consideration also invite reflections on assumptions and shared American values: In this case not necessarily (but also) assumptions about race/ethnicity, gender or class - the traditional three - but about the human body, its biology and our material surroundings. The representations range from accounts of DIY biology in newspapers and online magazines such as Wired, Mashable, or the New York Times; websites and product descriptions; popular science accounts and self-help books; to videos, films and documentaries. As this list makes clear, I am working with a broad understanding of texts and a wide variety of media. ${ }^{7}$ This variety is chosen consciously to demonstrate the range of possible texts individuals might encounter in public discussions. Let me provide a brief explanation for how these different types of sources are well suited to address some of the core issues of the DIY trend.

For one, most of them could be considered as classical 'pop-cultural' formats. Popular culture, in my opinion, is much better suited to capture on-going and timely cultural concerns than more avantgarde formats. They do not only reflect but also shape and feed dominant assumptions, stereotypes and imaginations. Victoria Pitts-Taylor argues that even if pop-cultural formats might be simplistic, exaggerated or misleading, they are still socially significant because "much of what people know about biomedical science comes from the press and from the experts in the self-help market" trying to reach their (lay) audience through a variety of (popular) media ("Plastic" 641). Audiences make sense of medicine and science also through the practices and products of culture - among which popular media are some of the fastest moving, most-consumed

6 Chapter 2.1 looks at the role of culture, and especially popular culture, for the sedimentation and translation of scientific knowledge, its relation to biology and role in the production of meaning from our shared biological materiality.

7 Krupar and Ehlers' methodology of biocultural studies suggests intertextual readings "between various kinds of cultural products, knowledges, policies and practices" to examine the separation between "discourse, practice and materiality" (7). 
channels. Popular media are one of the main avenues through which biomedical innovations and practices are communicated to lay audiences, many through some sort of science journalism or popular accounts of science. Popular accounts of science do not only perpetuate scientism as the dominant, culturally shared world-view but also show how fashionable science has become today (Erickson 174-75, 187-89). However, popular accounts of science are not just popular because they feed the public's desire to be informed but also because they are entertaining (Erickson 187-89). ${ }^{8}$ They have more leeway when presenting scientific facts than more esoteric forms of publication and popularization, such as scientific journals. Media accounts of DIY techniques and nonfiction science books are two ways in which science journalism enters my discussion, most prominently in Chapter 7 in my discussion of the DIY EpiPencil and its media representation (Case Study IV Make Your Own Medicine), the analysis of Wohlsen's portrayal of Kay Aull's famous genetic test hack in Chapter 8 (Case Study VI Make It Personal) and Chapter 10's discussion of Wohlsen's take on technological hacking (Case Study IX Cheap and Functional). A special sub-section of non-fiction (science) writing, self-help books, are the main subject of my analysis of biohacking 'manifestos' in Chapter 6 (Case Study I Be Smarter, Stronger, Happier). All these formats are especially relevant as forms of translation but also because from them we can infer common associations with DIY biology and its presumed relation to biotechnology more generally.

Because they fit so well into the contemporary audio-visual culture, documentaries are another popular and comprehensible medium that is used for the sedimentation of scientific 'facts' into the public sphere. Deborah Steinberg describes the documentary as a "genre of visual culture whose particular capital is 'truth'." As such it pretends to offer "unmediated facts" (132). Documentaries seem to be particularly appealing to contemporary audiences in the knowledge society, they are a quick and easy way of selfdetermined learning. My case studies make use of documentaries mainly as niche productions, such as the above quoted YouTube documentary about biohacking, but also in the form of popular shows, such as Netflix's Take Your Pills (2018), the object of interest in Case Study III Take Your Pills, Become Limitless in Chapter 7.

A more affective, desire- and need-based popular medium that I include in my discussion are advertisements and other forms of public communication, including websites, ads, and commercial videos. These are prominent sources in my discussion of Dave Asprey's Bulletproof brand and the new experts of the soma in Chapter 6 (Case Study II Biohacking on the Web), genetic testing companies' web presences in Chapter 8 (Case Study V "Welcome to You"), Chapter 9's discussion of technological integration through tracking and grinding (Case Study VII Quantifiably Me and Case Study VIII Implants, Grinders and Technological Immersion), as well as my consideration of tinkered medical tools in Chapter 10 (Case Study X Practical Solutions). Nelkin and Lindee argue that advertising images are significant indicators of public perception: They are made to sell ideas and products, are broadly understood and widely appreciated, and resonate

This type of "edutainment" makes the popularization through science journalists and writers a key component in the processes of translation and mediation of scientific knowledge, cf. Rose, Politics of Life 29-30. 
with consumer experience and popular belief (13). ${ }^{9}$ Fittingly, I use them to also elucidate some of the promises and affective atmospheres that are created around products and practices.

The practices and representations in my case studies, however, should not be considered a comprehensive list or extensive record. They are selective examples that I use as illustrations and sources for textual and discourse analysis. Many of them were found using Google searches with specific key terms or searches on web platforms like Amazon.com. ${ }^{10}$ Some I stumbled upon during my research, others are more widely known. This web-based approach was chosen to reflect the primary means through which an interested public finds these texts and practices - online, either on purpose or accidentally. It also aligns with the web-based focus of DIY biology and the maker movement more generally. I hope that the variety of sources presents a realistic view of what types of information, discourses and images an individual will encounter during their search for information or opportunities for participation. Each chapter first provides context and a brief introduction to the practices before looking closer at their representations. This way I hope to work out the specifics while not losing sight of common concerns. The order of chapters and case studies is not one of importance but rather tries to group some of these practices into separate but related clusters, which themselves are not always clear cut, but rather overlap and intersect.

Medical advertising is no exception: The USA are one of the few countries that allow regulated direct-to-consumer advertising for drugs. These do not only prompt patient-consumers to go 'shopping' for treatments, but they can also play a role in the conception of diseases and accepted experiences of illness. My MA thesis on the representation of Multiple Sclerosis, for example, has looked at the representation of people with MS in medical advertisements for MS drugs and found that most of them use a desire for (and promise of) fitness and activity, making the neurological disease all but invisible. As such, they might shape how affected individuals experience and evaluate their own embodiment, cf. Crewe-Salfeld.

10 Among those key terms are more generalized ones, such as biohacking, DIY biology, or DIY medicine, and more specific ones like grinding, genetic testing, or names of companies. 